\title{
CSM-Interacting Stripped-Envelope Supernovae
}

\author{
Massimo Turatto and Andrea Pastorello \\ Osservatorio Astronomico di Padova - INAF, \\ vicolo dell'Osservatorio 5, 35122, Padova, Italia \\ email: massimo.turatto@oapd.inaf.it
}

\begin{abstract}
Type Ibn supernovae (SNIbn) are a small group of core-collapse events whose ejecta interact with a He-rich and H-deprived circumstellar medium. The explosion of the prototype of this family, SN 2006jc, was heralded by a major stellar eruption two years before its core collapse. This unexpected discovery increased significantly the interest of the astronomical community toward these unusual events. A number of other objects have been included to the SNIbn zoo. In these contribution we review our current knowledge on this rare family of interacting, strippedenvelope supernovae (SE-SNe).
\end{abstract}

Keywords. supernovae: general, supernovae: SN 2006jc, SN 1999cq, SN 2000er, SN 2002ao, SN 2005la, SN 2010al, SN 2011hw, LSQ12btw, PS1-12sk, stars: winds

\section{Introduction}

Growing evidence has been recently accumulated for the presence of dense circumstellar media around supernova (SN) precursors. This is primarily true for core-collapse SNe descending from massive progenitors $\mathrm{M} \geqslant 8 \mathrm{M}_{\odot}$, though sometimes controversially claimed also for thermonuclear SNe (Hamuy et al. 2003, Benetti et al. 2006, Silverman et al. 2013). The most massive stars stars lose large fraction of their initial mass via intense winds or episodic giant eruptions that can remove up to tens of solar masses (about $10 \mathrm{M}_{\odot}$ in $\eta$ Car; Smith et al. 2003). Some type IIn SNe, whose observables are explained with the interaction of the SN ejecta with dense H-rich circum-stellar matter (CSM), have been associated to very massive progenitors such as LBV, though several theoretical issues remain unsolved. Also in the $25-40 \mathrm{M}_{\odot}$ initial mass range stars can lose large amounts of their outer envelopes before the final explosions via powerful winds. The SNe associated to progenitors stripped of their envelopes are called of type Ib, if deprived only of the $\mathrm{H}$ layer, or of type Ic, if deprived also of He (cfr. Turatto et al. 2007 and references therein). At lower masses $\left(\leqslant 25 \mathrm{M}_{\odot}\right)$ the stellar winds are less efficient and the stars reach the collapse of the core still retaining a significant fraction of the $\mathrm{H}$ envelope, and the corresponding SNe are called of type II. The amount of $\mathrm{H}$ left in the envelope plays a major role in the optical display, because $\mathrm{H}$ recombination supports long-lasting plateaus in the light curves (Pumo \& Zampieri 2011 and references therein).

The impact of SN ejecta on the slow material close to dying stars is revealed via radio, X-ray and optical emissions. The optical emission originates from the optically thin circumstellar matter ionized by the shock and the SN radiation field. The optical spectra are characterized by blue colours, narrow (a few $1000 \mathrm{~km} \mathrm{~s}^{-1}$ ) Balmer emission lines and slow spectral evolution. Objects with such features are usually called as of type IIn (cfr. Turatto et al. 2007). Recently, however, a small number of objects have have been discovered with similar blue colours but narrow $\mathrm{HeI}$ emissions and no, or very 
faint, $\mathrm{H}$ lines. In close analogy to type IIn these objects have been considered as SE SNe expanding in a dense He-rich CSM, and dubbed as type Ibn SNe(Pastorello et al. 2007).

Aim of this paper is to check if such scenario hold to the inclusion of the most recent members.

\section{Previous Wisdom}

Pastorello et al. 2008a analyzed synoptically the observations of type Ibn SNe available at the time, and found that they followed an overall similar photometric and spectroscopic evolution, though still having a number of distinctive peculiarities. The earliest epochs, available only for SN 2000er, showed a blue continuum with HeI lines having narrow $\left(\sim 800-900 \mathrm{~km} \mathrm{~s}^{-1}\right)$ P-Cyg absorptions, forming in the unshocked CSM, and broader $\left(\sim 5000 \mathrm{~km} \mathrm{~s}^{-1}\right)$ red wings, possibly arising in the SN ejecta (a sort of a SNIb expanding in a He-rich CSM).

The subsequent epochs up to late times were best studied in SN 2006jc which surprisingly showed a pre-discovery outbust about two years before the SN explosion (Pastorello et al. 2007). Remarkable was a near-UV/blue $(\lambda<5700 \AA)$ pseudo-continuum caused by a blend of broad fluorescent lines mainly of Fe (Foley et al. 2007). Together with broad (4000-9000 $\mathrm{km} \mathrm{s}^{-1}$ ) lines of intermediate mass elements in the SN ejecta, it showed moderately narrow (2000-3000 $\mathrm{km} \mathrm{s}^{-1}$ ) HeI emissions with narrower absorptions pointing out unshocked CSM at $\sim 1000 \mathrm{~km} \mathrm{~s}^{-1}$, typical of WR stars. At later phases $(\mathrm{t}>130 \mathrm{~d})$ the blue continuum faded away while narrow $\mathrm{H}$ emissions appeared indicating that finally the ejecta entered H-rich material (Pastorello et al. 2008a). Two other objects (SNe 1999cq and 2002ao) fitted well the evolution described above.

The light curves were relatively narrow, not dissimilar from those of other SE-SNe. All observations were reasonably well interpreted as the result of the explosion of a WR progenitor exploding in a dense He-rich CSM.

\section{Increasing the Sample}

After the initial unexpected discoveries, the newly announced SNIbn candidates have been studied in detail.

Probably the best studied object is SN 2010al, observed from the UV to the radio domain (ATEL 2532, ATEL 2513). Initially classified as a SNIIn similar to SN 1998S, because of the clear but non dominant presence of $\mathrm{H}$ (Cooke et al. 2010), the early blue spectra revealed narrow lines of HeI, HeII, NIII and CIII, typical of WR stars over a blue continuum (Pastorello et al. 2011, Pastorello et al. 2013a) with P-Cyg absorptions indicative of a CSM expanding at velocity $v \sim 1000 \mathrm{~km} \mathrm{~s}^{-1}$. One month later the spectra transformed to match those of SN 2006jc, with lines of intermediate mass elements with FWHM(IME) 5000 $\mathrm{km} \mathrm{s}^{-1}$.

If SN 2010al displayed weak $\mathrm{H}$ even at early stages, implying that the CSM lately ejected by the star had a non-negligible amount of $\mathrm{H}$, a further step in bridging the gap between type Ibn and IIn was made with SN 2005la and SN 2011hw. In the early spectra of the former, $\mathrm{H}$ lines had comparable strengths and widths as $\mathrm{HeI}\left(\sim 1000 \mathrm{~km} \mathrm{~s}^{-1}\right)$. These showed also P-Cyg absorptions, while IME lines, interpreted as representative of the ejecta, had FWHM $(I M E) \sim 4900 \mathrm{~km} \mathrm{~s}^{-1}$ (Pastorello et al. 2008b). The narrow P-Cyg absorptions disappeared soon and the line widths increased up to $v_{F W H M}=4200$ $\mathrm{km} \mathrm{s}^{-1}$, closely matching the IME velocity. The light curve was definitely broader than other SNIbn, with modulations possibly due to an increased CSM interaction. SN 2011hw 
(Smith et al. 2012, Pastorello et al. 2013b) was similar to SN 2005la with strong, moderately narrow $\left(v \sim 1900 \mathrm{~km} \mathrm{~s}^{-1}\right) \mathrm{HeI}$ and $\mathrm{H}$ from the very beginning, indicating that the CSM was not completely H-deprived. It also showed unresolved $\left(v<250 \mathrm{~km} \mathrm{~s}^{-1}\right)$ coronal lines (e.g. [ArX], [FeV], [FeVII]), observed in strongly interacting type IIn, e.g. 1988Z (Turatto et al. 1993), indicating that X-ray penetrates the slow, unshocked CSM. Also its light curve was flatter and clearly different from those of other SNIbn. In other words, differently from previously known SNIbn, these two objects revealed the presence of He- and H-rich material ejected short before the explosion.

Puzzling has been the discovery of PS1-12sk in the Brightest Cluster Galaxy CGCG208042 (Sanders et al. 2013). Overall similar to SN 2006jc, but for early evidence of H, it rose the issue of its parent population because of the elliptical morphology of the host. A detailed analysis of the possible scenario involving degenerate progenitors does not explain the observables (e.g. presence of $\mathrm{He}, \mathrm{H}$, dense CSM, ejected ${ }^{56} \mathrm{Ni}$ mass, etc.). Though there is no direct evidence of star formation at the site of explosion, the activity of the nucleus and the indication of cooling flows seem to indicate that PS1-12sk is one of the rare (but known) cases of CC-SN exploding in early type galaxies with signs of SF (Hakobyan et al. 2008).

The recent LSQ12btw, another victim of the current anarchy in naming SNe, adds little to the story. It showed the typical blue pseudo-continuum due to blends of FeII lines, relatively broad HeI $\left(v \sim 4000 \mathrm{~km} \mathrm{~s}^{-1}\right)$, and a fast evolving light curve (Pastorello et al. 2013b).

A few other objects might belong to this class, e.g. OGLE-2012-SN-006, but scanty material has been published so far.

\section{The updated Scenario}

Figure 1 has been drawn to sketch a scenario for type Ibn events, in the context of interacting SNe. The spectra are displayed according to a decreasing relative intensity of $\mathrm{H}$ CSM lines (form top to bottom).

At the top we show the spectrum of SN 2010jl, a SNIIn dominated by Balmer lines with multiple components. Weak He lines are present with comparable velocities. Interestingly, a luminous blue source has been detected at the position of the SN in pre-SN HST imaging. Whether this is really the SN progenitor itself or a young star cluster, the conclusion seems unavoidable that the progenitor had $\mathrm{M}>30 \mathrm{M}_{\odot}$ (Smith et al. 2011). Consistently, the spectral evolution indicates that the progenitor (possible a LBV) underwent a tremendous mass loss in the last decades before the explosion (Zhang et al. 2012). However, the presence of slow preshock winds ( $\mathrm{v}=28 \mathrm{~km} \mathrm{~s}^{-1}$, Smith et al. 2011) cannot be easy reconciled with the LBV scenario.

Below the SN 2010jl spectrum are reported those of SN 2005la and SN 2011hw. Their $\mathrm{H}$ and $\mathrm{He}$ lines are comparable in strength and width at all epochs, indicating that they arise from the same layers of shocked CSM located close to the exploding star. Lines of intermediate mass elements, present in the red parts of the spectra, are broader $(\mathrm{v} \sim 5000$ $\mathrm{km} \mathrm{s}^{-1}$ ) suggesting that they are forming in the SN ejecta. The presence of $\mathrm{H}$ has been invoked (Smith et al. 2012) to explain the existence of a $6000 \mathrm{~K}$ black-body which dilutes the typical near-UV/blue continuum, clearly visible in 2006jc-like objects, and reduces the contrast of the spectral features. Such $6000 \mathrm{~K}$ BB may contribute to sustain the light curves of the two SNe that appear broader than those of other He-rich interacting SNe. We are probably facing stars that ejected short before the explosions consistent amounts of material containing $\mathrm{He}$ and $\mathrm{H}$ and may be transitioning from the LBV to the WR stages (Smith et al. 2012, Pastorello et al. 2013a). 


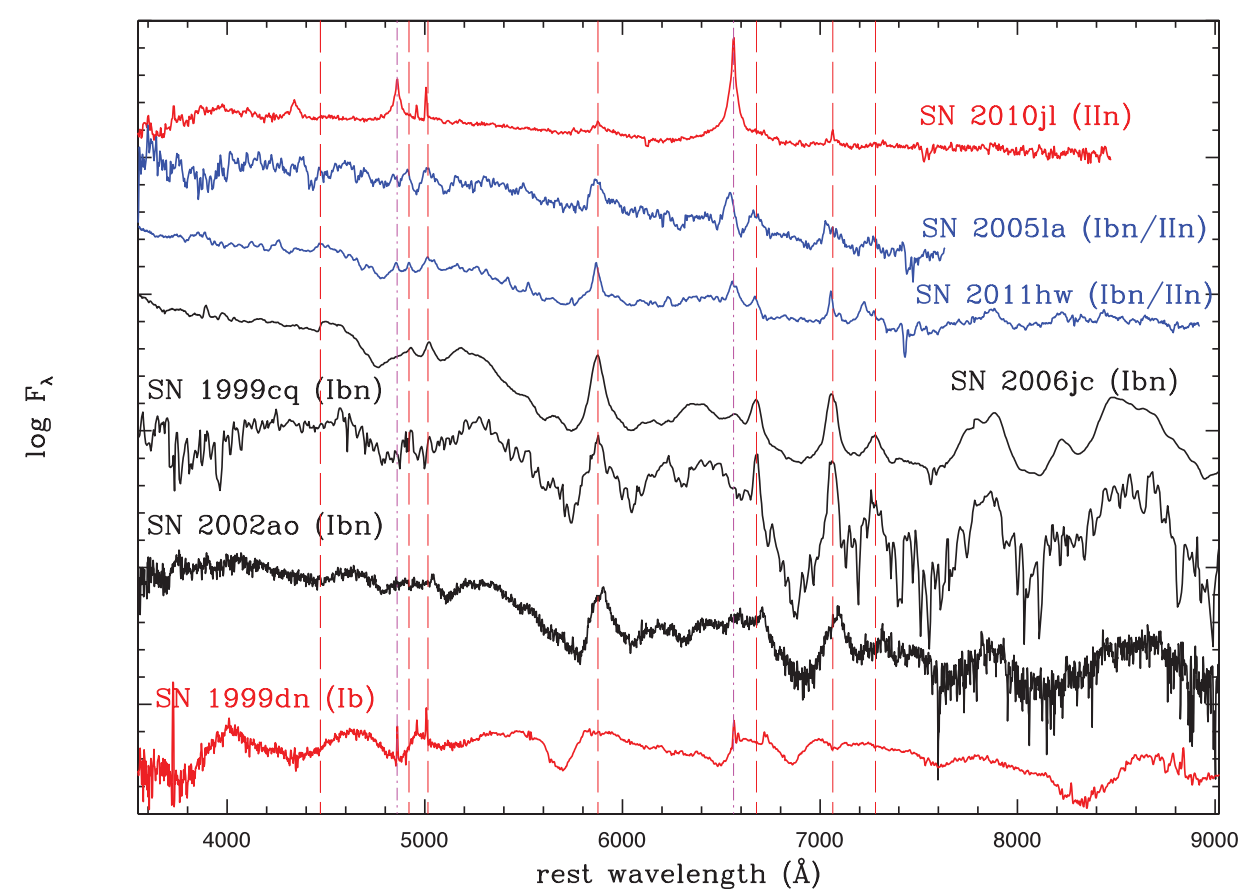

Figure 1. Comparison of spectra of different flavors of CSM interacting SNe, obtained at about 3-4 weeks after the estimated epoch of explosion. Vertical orange dashed lines mark the positions of the main He features, while red dot-dashed lines reveal the rest wavelength positions of $\mathrm{H} \alpha$ and $\mathrm{H} \beta$. At the top we show the spectrum of SN $2010 \mathrm{jj}$, strongly interacting with a dense H-rich CSM (IIn). Below are reported the spectra of SN 2005la and SN 2011hw, whose CSM seems enriched also He (IIn/Ibn). The CSM of the three subsequent SNe, 2006jc, 199cq and 2002ao, does not show $\mathrm{H}$ lines (Ibn). At the bottom is reported for comparison the spectrum of the normal SN 1999dn at comparable phase.

The middle-lower part of Fig. 1 shows spectra of three canonical SNIbn, 2006jc, 1999cq and 2002ao (Matheson et al. 2000, Pastorello et al. 2008a). Their spectra show: 1prominent $\mathrm{He}$ emission lines and weak evidence of $\mathrm{H}$ (if any), 2- strong nearUV/blue pseudo-continuum, 3- broad lines of IME, 4- evidence of fast $\left(\sim 1000 \mathrm{~km} \mathrm{~s}^{-1}\right)$ CSM. The light curves are narrow and resembling those of non-interacting type Ibc SNe. All characteristics are, therefore, well compatible with having WCO progenitors exploded in He-dominated CSM. The overall observed homogeneity of SNIbn is quite surprising for objects dominated by a pre-existing CSM whose geometry and density can be very diverse, and probably is indicative that the role of interaction is not dominant. At the very bottom is reported for comparison the spectrum of the prototypical type Ib SN 1999dn (Benetti et al. 2011). The comparison highlights as some of the major ondulations in the continuum of SNIbn are probably associated to an underlying type Ibc SN. All SNIbn exploded in spiral galaxies spanning a broad range of metallicities (Pastorello et al. 2013b), with the exception of PS1-12sk exploded in an elliptical with indications of SF.

We conclude, therefore, that the early scenario proposed to explain the observations of intermediate-width $\mathrm{He}$ and $\mathrm{H}$ features, holds, and that the observed properties of type Ibn are consistent with the explosion of massive WR or transitional LBV/WR precursors that recently enriched their CSM with He-rich material. 


\section{References}

Anupama, G. C., Sahu, D. K., Gurugubelli, U. K., Prabhu, T. P., Tominaga, N., Tanaka, M., \& Nomoto, K. 2009, MNRAS, 392, 894

Benetti, S., Cappellaro, E., Turatto, M., Taubenberger, S., Harutyunyan, A., \& Valenti, S., 2006, ApJ (Letters), 653, L129

Benetti, S., et al. 2011, MNRAS, 411, 2726

Cooke, J., Ellis, R. S., Nugent, P. E., Howell, D. A., Sullivan, M., \& Gal-Yam, A., 2010, ATEL, 2491

Foley, R. J., et al. 2007, ApJ, 657, L105

Hakobyan, A. A., Petrosian, A. R., McLean, B., Kunth, D., Allen, R. J., Turatto, M., \& Barbon, R., 2008, A \& A, 488, 523 (2008).

Hamuy, M., et al. 2003, Nature, 424, 651

Matheson, T., et al. 2000, ApJ, 119, 2303

Mattila, S., et al. 2008, MNRAS, 389, 141

Pastorello, A., et al. 2007, Nature, 447, 829

Pastorello, A., et al. 2008a, MNRAS, 389, 113

Pastorello, A., et al. 2008b, MNRAS, 389, 131

Pastorello, A., Benetti, S., Bufano, F., Kankare, E., Mattila, S., Turatto, M., \& Cupani, G. 2011, $A N, 332,266$

Pastorello, A., et al. 2012, in preparation

Pastorello, A., et al. 2012, in preparation

Pumo, M. L. \& Zampieri, L. 2011, ApJ, 741, 41

Sanders, N. E., et al. 2013, ApJ submitted (eprint arXiv:1303.1818)

Silverman, J. M., et al. 2013, ApJ, submitted (eprint arXiv:1304.0763)

Smith, N., et al. 2003, AJ, 125, 1458

Smith, N., et al. 2011, ApJ, 732, 63

Smith, N., et al. 2012, MNRAS, 426, 1905

Turatto, M., Cappellaro, E., Danziger, I. J., Benetti, S., Gouiffes, C., \& Della Valle, M., 1993, MNRAS, 262, 128

Turatto, M., Benetti, S., \& Pastorello, A., 2007, in: S. Immler \& K. Weiler (eds.), AIP Conference Proceedings 937, p. 187

Zhang, T., et al. ApJ, 144, 131

\section{Discussion}

Chevalier: The light curves are bright; is this due to interaction or high $56 \mathrm{Ni}$ ?

TuRATto: The light curves of SNIbn are moderately bright $\left(L_{b o l} \sim 10^{43} \mathrm{erg} / \mathrm{s}\right) \mathrm{Ni}$ masses have been estimated only in few objects because light curves are in most cases not sufficiently extended. For SN 2006jc the estimated Ni mass is $M_{N i}=0.250 .40$ (Pastorello 2008). Most probably the interaction acts flattening the light curve.

MAEDA: Do you see any signature of the dust formation in SNe other than SN 2006jc? For example, when you have late-time spectra (e.g. $\geqslant 2$ months), do you see the blue-shift in lines?

TuRTTo: The objects identified and studied so far are relatively distant. Spectral observations, therefore, are limited in most cases to the first 2 months best in SN 2006jc, in which dust evidence emerged just at about day 50. I am not aware of the results of the observations of 2010 al at late time. 